\title{
SPATIAL OFFSETS OF INTERPLANETARY ION AND ELECTRON SOURCE REGIONS
}

\author{
E. E. Chollet ${ }^{1,5}$, J. Giacalone ${ }^{2}$, R. M. Skoug ${ }^{3}$, J. T. Steinberg ${ }^{3}$, and J. T. Gosling ${ }^{4}$ \\ ${ }^{1}$ Space Radiation Laboratory, California Institute of Technology, Pasadena, CA 91125, USA \\ ${ }^{2}$ Lunar and Planetary Laboratory, University of Arizona, Tucson, AZ 85721, USA \\ ${ }^{3}$ Los Alamos National Laboratory, Los Alamos, NM 87545, USA \\ ${ }^{4}$ Laboratory for Atmospheric and Space Physics, University of Colorado, Boulder, CO 80303, USA \\ Received 2009 July 16; accepted 2009 September 29; published 2009 October 22
}

\begin{abstract}
Observations of impulsive solar energetic particles provide unique insight into the process of particle acceleration in the solar atmosphere. X-ray and $\gamma$-ray observations of precipitating particles from solar flares have shown offsets between the ion and the electron emission. We present Advanced Composition Explorer (ACE) observations of interplanetary energetic ion and suprathermal electron events with intensity variations related to connection or lack thereof to the particle source. These results indicate an offset between the acceleration regions of ions and electrons of the same order of magnitude as that observed in the $\gamma$-ray events. However, if the particle event originates at the magnetic footpoints of an interplanetary coronal mass ejection, the ACE observations do not exhibit this offset, suggesting that different magnetic geometry or acceleration processes may be present in those regions.
\end{abstract}

Key words: acceleration of particles - interplanetary medium - Sun: magnetic fields - Sun: particle emission

Online-only material: color figures

\section{INTRODUCTION}

Though acceleration of charged particles is ubiquitous in the universe, a detailed understanding of the mechanisms involved remains a great puzzle. Solar flares offer one of the best natural laboratories for discerning these mechanisms, because of their proximity and frequency as well as the wide range of instruments available to study them. It is generally thought that, in solar flares, stored magnetic energy is released through reconnection and accelerates particles in the solar corona to high speeds (Lin \& Hudson 1976; Priest \& Forbes 2002). These particles either impact the solar chromosphere or escape into interplanetary space. Electrons that impact the solar atmosphere produce bremsstrahlung X-rays, and ions produce nuclearcollision $\gamma$-rays, including neutron capture lines. The RHESSI spacecraft (Lin et al. 2002) was launched in 2002 with the goal of studying acceleration mechanisms through observations of these $\mathrm{X}$ - and $\gamma$-rays. Accelerated particles that escape the Sun travel out through the solar wind following magnetic field lines. If a spacecraft such as the Advanced Composition Explorer (ACE; Stone et al. 1998) intersects a field line connected to a particle source, it observes the accelerated particles as well, and comparisons between the in situ and X-ray observations provide a multi-point observation of the acceleration region.

Frequently, the in situ observations of flare-related energetic particle events show sharp increases and drops in intensity simultaneously across all energies, as the spacecraft alternately connects to field lines containing energetic particles and those lacking them. In flare-related energetic particle events (often called "impulsive" events), particles are accelerated close to the Sun and injected onto field lines essentially all at one instant. These events are easily distinguished from other kinds of energetic particle events in situ through their composition enhancements in ${ }^{3} \mathrm{He}$ and heavy species such as iron. They are also typically accompanied by Type III radio bursts, which are excited as the flare-accelerated electrons streams out into the inter-

\footnotetext{
5 Previously at the Lunar and Planetary Laboratory, University of Arizona, Tucson, AZ 85721, USA.
}

planetary medium. Early studies (Anderson \& Dougherty 1986; Buttighoffer 1988; Anderson et al. 1992) reported "channels" in flare-related electron events and square wave-like decreases in intensity, and they suggested the channels were related to the structure of the background magnetic field. Since the launch of $A C E$, intensity "dropouts" have been reported in ions with energies between $20 \mathrm{keV}$ nucleon ${ }^{-1}$ and $10 \mathrm{MeV}$ nucleon ${ }^{-1}$ (Mazur et al. 2000; Giacalone et al. 2000) observed with the Ultra-LowEnergy Isotope Spectrometer (ULEIS; Mason et al. 1998) and in suprathermal electrons (Gosling et al. 2004a, 2004b) with energies between 73 and $1370 \mathrm{eV}$ with the Solar Wind Electron Proton Alpha Monitor (SWEPAM; McComas et al. 1998). Since intensity drops are caused by lack of connection to the particle acceleration region, they are a powerful tool for testing particle acceleration theories. For example, several studies (Chollet \& Giacalone 2008; Giacalone et al. 2000) have discussed how the frequency of dispersionless intensity variations can be used to make inferences about the sizes of particle acceleration regions, since a large source will fill all field lines observed by the spacecraft, but a small source will fill very few.

Recent remote sensing results have suggested that ions and electrons may be accelerated in different locations. Observations of large flares by the RHESSI spacecraft have shown puzzling offsets between the centroids of electron-produced X-rays and ion-produced $\gamma$-rays on the order of tens of thousands of kilometers (Hurford et al. 2006, 2003; Lin et al. 2003). Though these offsets could be produced by separation of species between acceleration and photon production by a large-scale electric field, the creation and maintenance of such a field in a region where particles are moving quickly and relatively freely seems impractical. If the particles are accelerated in different regions, they should naturally follow different magnetic field lines to their eventual impact in the higher density chromosphere, producing the observed offset in X-rays and $\gamma$-rays. Similarly, the portion of particles traveling away from the Sun should be injected onto different field lines, resulting in field lines which are connected to the ion region but not to the electron region and vice versa. $A C E$ will then see at one particular instant in time a 
dropout or increase in one species but not the other, as shown in some of the case studies of Gosling et al. (2004a).

In this work, we perform a detailed comparison of electron and ion acceleration regions through the study of intensity variations in interplanetary particle events. In Section 2, we describe the spacecraft and instruments along with the sample selection method. We compare the results for each event in Section 3, and we use these observations to make inferences about the ion and electron source regions.

\section{OBSERVATIONS}

The data studied here were obtained by two instruments aboard the $A C E$ spacecraft: the ion data from ULEIS and the electron data from SWEPAM. ACE was launched in 1997 to orbit around the L1 Lagrange point where it is always in the solar wind. We use electron data from SWEPAM in 10 logarithmically spaced energy bins from 73.3 to $1370 \mathrm{eV}$ (summing over pitch angle). We use ion data from ULEIS with masses between 12 and $60 \mathrm{AMU}$ (carbon through iron peak) and energies between $50 \mathrm{keV}$ nucleon $^{-1}$ and $10 \mathrm{MeV}$ nucleon $^{-1}$.

Transport effects and temporal evolution of the source must be considered when using interplanetary data to study source regions. The particles are injected onto field lines impulsively at the Sun, but, because of their different speeds, the spacecraft observes a velocity dispersion at $1 \mathrm{AU}$. Since the dropouts are related to a lack of connection to the source, they are dispersionless and can easily be separated from the transportrelated dispersion. Using different species with the same particle speed reduces the contribution of temporal evolution of the source to the differences between fluctuations in the ion and electron data. A $73.3 \mathrm{eV}$ electron has an inverse speed of $8.2 \mathrm{hr} \mathrm{AU}^{-1}$, and a $1370 \mathrm{eV}$ electron has an inverse speed of $1.9 \mathrm{hr} \mathrm{AU}^{-1}$. The ion data used here range roughly between 1 and $17 \mathrm{hr} \mathrm{AU}{ }^{-1}$, so the ULEIS and SWEPAM data have significantly overlapping speed ranges, as noted by Gosling et al. (2004a).

We have selected flare-related ion events observed by the $A C E$ spacecraft between late 1997 and 2006. The events used are drawn from the work of Chollet \& Giacalone (2008). In that study, they chose events using standard criteria for flare-related energetic particle events: clear velocity dispersions and elevated ${ }^{3} \mathrm{He}$ and $\mathrm{Fe}$. In addition, the events selected had relatively high intensities, making the dropout field lines more distinguishable from the field lines that were connected to the particle source. Variations were determined to be present by testing if the intensity changed by $15 \%$ or more in a sliding window with a 0.2 day width stepped to 0.1 days. The reader should refer to that paper for more details about the sample selection and the selection of events with variations. In this study, we have only used the events with variations, so the sample (listed in Table 1) consists of 32 events.

Events in category I show simultaneous dropouts and increases in the electrons and ions, while events in category II show dropouts and increases at different times. Two events illustrating category I and category II are presented in Figure 1. We only include the energies where the electron burst was observed, and the speed range covered by those electron channels is marked with blue horizontal lines on the ion plot (bottom plot). Dispersionless changes in intensity are marked with red vertical lines. The 2000 August event (category I) had a clear velocity dispersion at onset in both the ions and the electrons, and dispersionless dropouts are clearly visible in both species centered at 17:15, 17:55, 18:20, 22:30, and 23:15 UT. By contrast, in the 1999 May event (category II), both species showed
Table 1

The Events Used in this Work

\begin{tabular}{|c|c|c|c|c|}
\hline Year & Time (Start) & Time (End) & Inside CME? $^{\mathrm{a}}$ & Coincident Variations? \\
\hline 1997 & 11/04 07:00 & $11 / 0514: 00$ & 0 & I \\
\hline 1997 & 11/14 19:00 & $11 / 1612: 00$ & 0 & II \\
\hline 1997 & $11 / 2217: 00$ & $11 / 2305: 00$ & 0 & I \\
\hline 1998 & 04/11 17:00 & $04 / 1222: 00$ & 1 & I \\
\hline 1998 & 05/09 07:00 & $05 / 1112: 00$ & 0 & I \\
\hline 1998 & 05/16 05:00 & $05 / 16$ 17:00 & 0 & IV \\
\hline 1998 & $07 / 1112: 00$ & 07/13 07:00 & 1 & I \\
\hline 1998 & 09/08 17:00 & 09/09 10:00 & 0 & I \\
\hline 1999 & 05/12 07:00 & 05/12 19:00 & 0 & II \\
\hline 1999 & $05 / 3014: 00$ & $05 / 3114: 00$ & 0 & III \\
\hline 1999 & 06/18 19:00 & $06 / 2014: 00$ & 0 & II \\
\hline 1999 & 08/07 22:00 & 08/09 00:00 & 0 & III \\
\hline 2000 & 04/01 22:00 & 04/03 05:00 & 0 & I \\
\hline 2000 & $04 / 27$ 17:00 & $04 / 2912: 00$ & 0 & IV \\
\hline 2000 & 05/01 10:00 & $05 / 0212: 00$ & 0 & II \\
\hline 2000 & 06/04 07:00 & 06/05 00:00 & 0 & IV \\
\hline 2000 & 08/12 10:00 & 08/13 05:00 & 1 & I \\
\hline 2000 & 11/07 19:00 & 11/09 12:00 & 1 & III \\
\hline 2000 & 12/28 00:00 & $12 / 2912: 00$ & 0 & IV \\
\hline 2001 & 04/03 00:00 & 04/04 12:00 & 1 & I \\
\hline 2001 & $06 / 2017: 00$ & $06 / 21$ 17:00 & 0 & II \\
\hline 2002 & $02 / 2002: 00$ & $02 / 2212: 00$ & 0 & IV \\
\hline 2002 & $04 / 1512: 00$ & 04/16 00:00 & 1 & I \\
\hline 2002 & 08/01 10:00 & 08/01 22:00 & 1 & I \\
\hline 2002 & 09/27 00:00 & 09/28 00:00 & 1 & I \\
\hline 2003 & 01/12 19:00 & 01/14 05:00 & 0 & I \\
\hline 2003 & 03/02 19:00 & 03/03 17:00 & 0 & III \\
\hline 2003 & 04/28 07:00 & 04/29 00:00 & 0 & II \\
\hline 2003 & 09/30 00:00 & 10/03 00:00 & 0 & III \\
\hline 2004 & 03/06 07:00 & 03/07 14:00 & 0 & II \\
\hline 2004 & 06/27 05:00 & 06/28 05:00 & 0 & I \\
\hline 2006 & $11 / 2110: 00$ & 11/22 07:00 & 0 & II \\
\hline
\end{tabular}

Notes.

a A 0 means no CME present, a 1 means a CME was present.

${ }^{\mathrm{b}}$ Category III means the event was unusable. Category IV means the variations were caused by a solar wind boundary, not source connections. Category II means $e^{-}$different from ions, and category I means $e^{-}$same as ions.

clear velocity dispersion at the beginning of the event, but the dispersionless features in the ions and the electrons were not simultaneous. The electrons had a dispersionless increase at 12:45 UT and a dropout centered at 14:50 UT, while the ions had a dispersionless increase at 11:15 UT and dispersionless dropouts centered at 13:30 and 15:50 UT.

Events with a category III or IV in Column 4 of Table 1 were not used in the final statistics. The five category IV events had dropouts or increases related to boundaries in the solar wind. Some of these boundaries (such as stream interfaces and current sheets) represent an abrupt shift in magnetic connection to a location in the corona far from the particle injection region (Gosling et al. 2004a). Two of the category IV events lack electron heat flux, suggesting the spacecraft is observing field lines disconnected from the Sun. Including events with only these types of variations would skew the overall statistics toward simultaneous ion and electron variations (Category I, see below) as compared to only including events where the magnetic footpoints stay in the vicinity of the flare. Finally, one category IV event coincides with the passage of a shock, and the change in particle intensity is caused by the shock passage, not by source connection. The five category III events either had their ion dropouts in the velocity range not covered by the SWEPAM instrument or many overlapping dispersions. If 

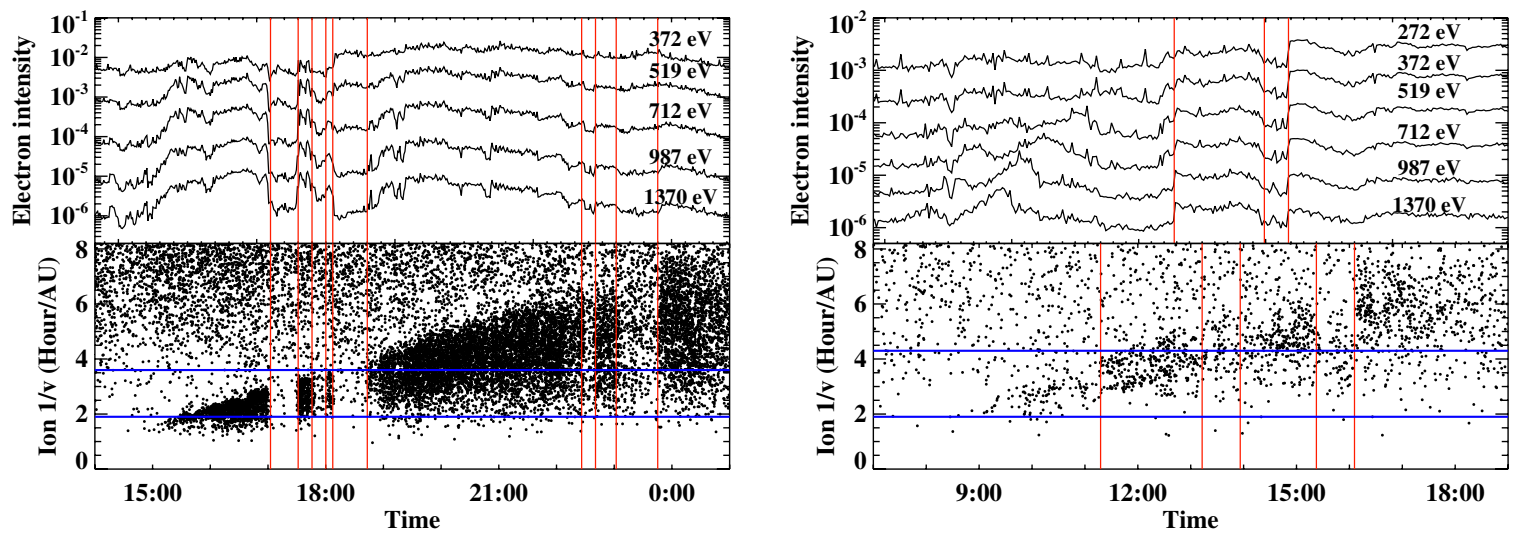

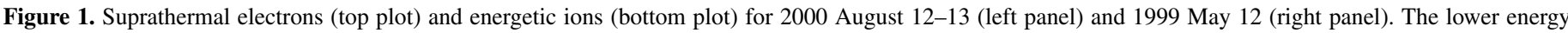

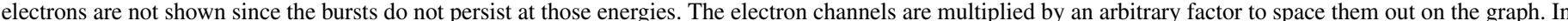

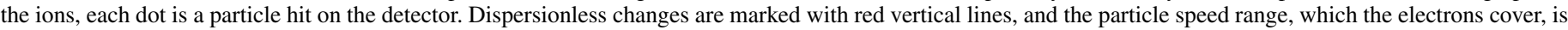
marked with horizontal blue lines in the bottom plot.

(A color version of this figure is available in the online journal.)

there are multiple particle injections in a short period, several velocity dispersions will be observed in the ion data at $1 \mathrm{AU}$. The particles from the different injections will arrive at $1 \mathrm{AU}$ at slightly different times, and dropouts or increases can occur in some dispersions in these multi-dispersion events but not others, resulting in dropouts being masked or spurious increases appearing. Also, the electrons are measured at 10 discrete energies while the ion energies are measured in a continuous way, so variations are more difficult to pick out in the electron data with multiple dispersions present. Thus, the present study covers only 22 events with category I or II in Table 1.

Energetic ions and suprathermal electrons accelerated in flares can travel up the magnetic field lines forming the legs of interplanetary coronal mass ejections (ICMEs) in a few hours with typical measured travel distances of 2-3 AU (e.g., Chollet et al. 2007; Larson et al. 1997). Since the ICMEs move much more slowly (requiring several days to reach $1 \mathrm{AU}$ ), they act as a background medium through which the energetic particles travel. We note that 8 of the 22 particle events in the sample occurred during passage of an ICME past the spacecraft. We have marked these events with a 1 in Column 3 of Table 1 , while events that did not occur with an ICME are marked with a 0 . We consider the two categories separately since we cannot assume a priori that the source regions for each type have the same magnetic geometry.

\section{ANALYSIS}

Category I and II events can each have multiple interpretations, but the groupings are based on the parts of the source regions to which the field line connects. In category I events (illustrated in Figure 2), all field lines are connected to a region where both ions and electrons have been injected or to a region where no particles were injected. At $t_{1}$ and $t_{3}$, the spacecraft detects no particle flux, while at $t_{2}$ and $t_{4}$, the spacecraft detects particle flux. Though there may be regions where one species is injected but not the other (as in Figure 2(B)), the spacecraft is never magnetically connected to those regions. Category II events include some period during the event where the spacecraft is connected to the source region for one species but not the other. The spacecraft may sometimes be connected to a region where both species are injected (Figure 3(A)), may be only connected to one species' source at a time (Figure 3(B)) or may

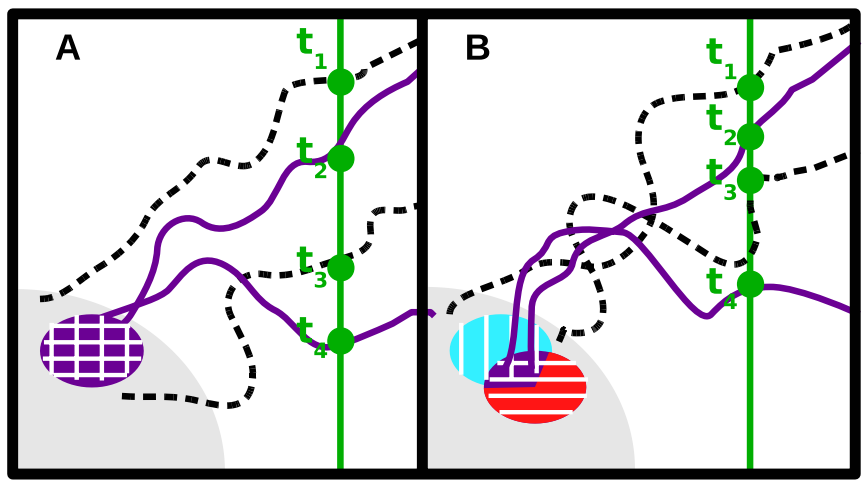

Figure 2. Cartoon illustrating potential scenarios producing category I events The light blue, vertical-striped area represents the electron injection region, the red horizontal-striped area represents the ion injection region, and the cross-hatched purple region represents where ions and electrons are both being injected. Black, dashed lines are magnetic field lines not connected to the particle sources, and solid lines contain particles with the colors corresponding to the source region colors. At times $t_{2}$ and $t_{4}$, the spacecraft (green track) observes both energetic ions and electrons, while at $t_{3}$ it observes dropouts.

(A color version of this figure is available in the online journal.)

only be connected to the ion source region and never the electron source region (Figure 3(C)). In the latter case, we do not have direct evidence that an electron source was present at all. However, we note that electron bursts related to impulsive flares are much more common than ion events (Gosling et al. 2004b). All the ion events in this sample with no apparent electron burst were accompanied by a Type III radio burst observed by the WAVES instrument on the nearby Wind spacecraft (Bougeret et al. 1995; Acuña et al. 1995). Since Type III bursts are produced by interplanetary electron bursts, it seems likely that an electron source was present but the $A C E$ spacecraft does not connect to it by chance.

The injection region geometry inferred from the X-ray data seems to be reflected in the interplanetary events when no ICME is present. Of the 14 events in the solar wind with no ICME present, eight of the events fell into category II and 6 into category I. If the ion and electron source regions were perfectly overlapping (as in Figure 2(A)), the sample would have no category II events. Similarly, if the ion and electron source regions never overlapped (as in Figure 3(B)), the sample 


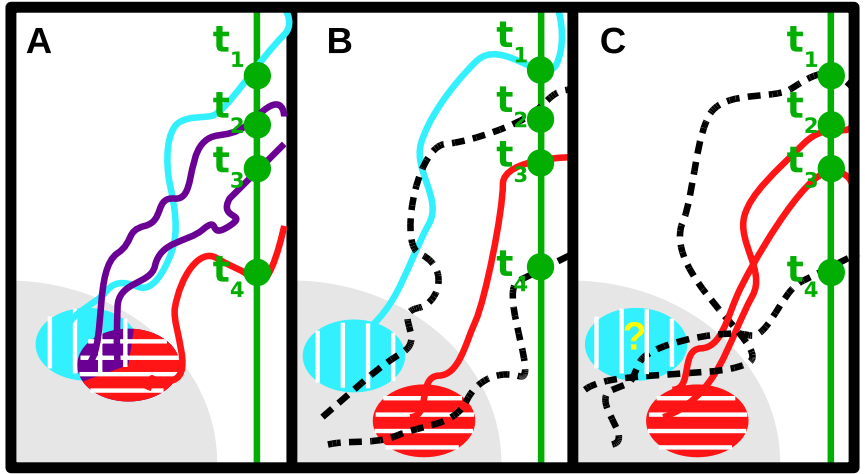

Figure 3. Cartoon illustrating potential scenarios producing category II events. The light blue, vertical-striped area represents the electron injection region, the red horizontal-striped area represents the ion injection region, and the cross-hatched purple region represents where ions and electrons are both being injected. Black, dashed lines are magnetic field lines not connected to the particle sources, and solid lines contain particles with the colors corresponding to the source region colors. In category II events, the spacecraft may see single and multiple-species field lines (left panel), both single-species field lines (middle panel) or just ion field lines (right panel).

(A color version of this figure is available in the online journal.)

would have no category I events. Though small number statistics may be at work, a roughly even split of category I and II events suggests that the source regions for the two species partially overlap and partially do not, i.e., their centroids are offset, as in Figures 2(B) and 3(A), and as are seen in the RHESSI data (Lin et al. 2003).

Following a simple field line meandering model, the observed offset in X-ray and $\gamma$-ray producing regions are consistent with the interplanetary particle results found in this study. Field line meandering occurs as the footpoints of field lines are moved around by supergranular motions at the Sun, with a typical rms speed of $0.5 \mathrm{~km} \mathrm{~s}^{-1}$ (Wang 1988). In a category II event like that in Figure 1, right panel, the spacecraft observes field lines connected to the electron source but not the ion source (around 13:30 UT) and field lines connected to the ion source but not the electron source (14:30 UT). To be observed so close together in time at $1 \mathrm{AU}$, these two field lines must have been adjoining when they left the Sun (with an assumed $400 \mathrm{~km} \mathrm{~s}^{-1}$ solar wind, approximately four days previously). During those four days, the field line footpoints at the Sun can separate due to random motion by a distance of $10^{5} \mathrm{~km}$ to an order of magnitude. The particles are then injected at these footpoints and fill the field lines in a few hours. The ion-only region to which the 14:30 field line connects and the electron-only region to which the 13:30 field line connects must therefore be separated by $\leqslant 10^{5} \mathrm{~km}$. The observed offset in the 2002 July 23 event was only $14,000 \mathrm{~km}$ (Lin et al. 2003), so it falls inside this limit. If this offset is typical for impulsive flares, it could easily produce the interplanetary result reported here.

All eight events observed in ICMEs fell in category I, suggesting that the source regions for these events may have different geometries from those with no ICMEs present. Figure 1, left panel, is an example of one of these ICME events, showing clear, matching dispersionless dropouts in the ions and electrons. Though the sample is small, this may suggest that the entire acceleration region accelerates both ions and electrons (as in Figure 2(A)) or that different acceleration mechanisms are at work when ICMEs are present. Since the magnetic topology at the base of an ICME is likely substantially different than that in a flare with no ICME present, the difference in results from the non-ICME interplanetary events is not totally unexpected. However, this result does suggest that theories of particle acceleration in flares should consider events at the footpoints of ICMEs separately from those with no ICME present.

\section{CONCLUSIONS}

We have presented interplanetary particle evidence that suggests that the acceleration regions for energetic ions and electrons are offset from one another. The observed offset in interplanetary particles is similar to the observed offset in X-ray and $\gamma$-ray emission from particle acceleration regions. However, the observed differences between ion and electron events disappear for interplanetary events inside ICMEs, so source region geometry or acceleration mechanism for these events may be substantially different from those with no ICME present. Though the number of events used here is small, as the solar cycle heads toward activity maximum, many more energetic particle events should be observed. Further confirmation of the interplanetary particle results and additional X-ray observations would be valuable to confirm the magnitude of the offset between the ion and electron acceleration regions and how often it occurs.

Much of this work was performed at the University of Arizona, as part of the first author's doctoral work. This work was supported, in part, by the NSF under grant ATM0447354.

\section{REFERENCES}

Acuña, M. H., Ogilvie, K. W., Baker, D. N., Curtis, S. A., Fairfield, D. H., \& Mish, W. H. 1995, Space Sci. Rev., 71, 5

Anderson, K. A., Chaizy, P., Lin, R. P., \& Sommers, J. 1992, Geophys. Res. Lett., 19,1283

Anderson, K. A., \& Dougherty, W. M. 1986, Sol. Phys., 103, 165

Bougeret, J.-L., et al. 1995, Space Sci. Rev., 71, 231

Buttighoffer, A. 1998, A\&A, 335, 295

Chollet, E. E., \& Giacalone, J. 2008, ApJ, 688, 1368

Chollet, E. E., Giacalone, J., Mazur, J. E., \& Al Dayeh, M. 2007, ApJ, 669, 615

Giacalone, J., Jokipii, J. R., \& Mazur, J. E. 2000, ApJ, 532, L75

Gosling, J. T., de Koening, C. A., Skoug, R. M., Steinberg, J. T., \& McComas, D. J. 2004b, J. Geophys. Res., 109, A05102

Gosling, J. T., Skoug, R. M., McComas, D. J., \& Mazur, J. E. 2004a, ApJ, 614, 412

Hurford, G. J., Krucker, S., Lin, R. P., Schwartz, R. A., Share, G. H., \& Smith, D. M. 2006, ApJ, 644, L93

Hurford, G. J., Schwartz, R. A., Krucker, S., Lin, R. P., Smith, D. M., \& Vilmer, N. 2003, ApJ, 595, L77

Larson, D. E., et al. 1997, Geophys. Res. Lett., 24, 1911

Lin, R. P., \& Hudson, H. S. 1976, Sol. Phys., 50, 153

Lin, R. P., et al. 2002, Sol. Phys., 210, 3

Lin, R. P., et al. 2003, ApJ, 595, L69

Mason, G. M., et al. 1998, Space Sci. Rev., 86, 409

Mazur, J. E., Mason, G. M., Dwyer, J. R., Giacalone, J., Jokipii, J. R., \& Stone, E. C. 2000, ApJ, 532, L79

McComas, D. J., Bame, S. J., Barker, P., Feldman, W. C., Phillips, J. L., Riley, P., \& Griffee, J. W. 1998, Space Sci. Rev., 86, 563

Priest, E. R., \& Forbes, T. G. 2002, A\&AR, 10, 313

Stone, E. C., Frandsen, A. M., Mewaldt, R. A., Christian, E. R., Margolies, D., Ormes, J. F., \& Snow, F. 1998, Space Sci. Rev., 86, 1

Wang, H. 1988, Sol. Phys., 116, 1 